ACCEPTED MANUSCRIPT

\title{
Kondo effect under the influence of spin-orbit coupling in a quantum wire
}

To cite this article before publication: Victor Lopes et al $2020 \mathrm{~J}$. Phys.: Condens. Matter in press https://doi.org/10.1088/1361-648X/aba45c

\section{Manuscript version: Accepted Manuscript}

Accepted Manuscript is "the version of the article accepted for publication including all changes made as a result of the peer review process, and which may also include the addition to the article by IOP Publishing of a header, an article ID, a cover sheet and/or an 'Accepted Manuscript' watermark, but excluding any other editing, typesetting or other changes made by IOP Publishing and/or its licensors"

This Accepted Manuscript is $@ 2020$ IOP Publishing Ltd.

During the embargo period (the 12 month period from the publication of the Version of Record of this article), the Accepted Manuscript is fully protected by copyright and cannot be reused or reposted elsewhere.

As the Version of Record of this article is going to be / has been published on a subscription basis, this Accepted Manuscript is available for reuse under a CC BY-NC-ND 3.0 licence after the 12 month embargo period.

After the embargo period, everyone is permitted to use copy and redistribute this article for non-commercial purposes only, provided that they adhere to all the terms of the licence https://creativecommons.org/licences/by-nc-nd/3.0

Although reasonable endeavours have been taken to obtain all necessary permissions from third parties to include their copyrighted content within this article, their full citation and copyright line may not be present in this Accepted Manuscript version. Before using any content from this article, please refer to the Version of Record on IOPscience once published for full citation and copyright details, as permissions will likely be required. All third party content is fully copyright protected, unless specifically stated otherwise in the figure caption in the Version of Record.

View the article online for updates and enhancements. 


\section{Lopes ${ }^{1,2}$, G. B. Martins ${ }^{3}$, M. Manya ${ }^{4}$, E. V. Anda ${ }^{1}$}

\section{Kondo effect under the influence of spin-orbit coupling in a quantum wire}

1 Departamento de Física, Pontifícia Universidade Católica do Rio de Janeiro (PUC-Rio), Rio de Janeiro, Rio de Janeiro, 22453-900, Brazil

${ }^{2}$ Departamento de Física Aplicada, Universidad de Alicante, San Vicente del Raspeig, 03690, Alicante, Spain

3 Instituto de Física, Universidade Federal de Uberlândia, Uberlândia, Minas Gerais, 38400-902, Brazil

${ }^{4}$ Instituto de Física, Universidade Federal Fluminense, 24210-346 Niterói, RJ, Brazil

E-mail: gbmartins@ufu.br

E-mail: enriquevictoranda@gmail.com

Abstract. The analysis of the impact of spin-orbit coupling (SOC) on the Kondo state has generated considerable controversy, mainly regarding the dependence of the Kondo temperature $T_{K}$ on SOC strength. Here, we study the one-dimensional (1D) single impurity Anderson model (SIAM) subjected to Rashba $(\alpha)$ and Dresselhaus $(\beta)$ SOC. It is shown that, due to time-reversal symmetry, the hybridization function between impurity and quantum wire is diagonal and spin independent (as it is the case for the zero-SOC SIAM), thus the finite-SOC SIAM has a Kondo ground state similar to that for the zero-SOC SIAM. This similarity allows the use of the Haldane expression for $T_{K}$, with parameters renormalized by SOC, which are calculated through a physically motivated change of basis. Analytic results for the parameters of the SOCrenormalized Haldane expression are obtained, facilitating the analysis of the SOC effect over $T_{K}$. It is found that SOC acting in the quantum wire exponentially decreases $T_{K}$ while SOC at the impurity exponentially increases it. These analytical results are fully supported by calculations using the Numerical Renormalization Group (NRG), applied to the wide-band regime, and the Projector Operator Approach, applied to the infinite- $U$ regime. Literature results, using Quantum Monte Carlo, for a system with Fermi energy near the bottom of the band, are qualitatively reproduced, using NRG. In addition, it is shown that the 1D SOC SIAM for arbitrary $\alpha$ and $\beta$ displays a persistent spin helix $\mathrm{SU}(2)$ symmetry similar to the one for a $2 \mathrm{D}$ Fermi sea with the restriction $\alpha=\beta$. 
Kondo effect under the influence of spin-orbit coupling in a quantum wire

Submitted to: J. Phys.: Condens. Matter

\section{Introduction}

It is well-known that magnetic impurities, when diluted in small concentrations in a metallic host, impart singular characteristics to its properties, most notably its resistivity, which acquires a minimum as a function of temperature [1]. This phenomenon, first observed in the mid 1930s [2], was given a satisfactory explanation only in 1964 by Jun Kondo [3]: the localized magnetic moment of the impurity is screened by the spins of the conduction electrons below a characteristic temperature, the so-called Kondo temperature $T_{K}$.

Recently, there has been much interest in the study of the Kondo regime of a magnetic impurity coupled to a metallic substrate presenting SOC. Theoretical work by Meir and Wingreen in 1994 [4] concluded that SOC does not suppress the Kondo effect. This subject lay dormant for many years, until a surge in interest (see Refs. 5,6) was stimulated by SOC's importance for systems with spintronic applications, e.g., the Datta-Das spin transistor [7]. Thus, SOC's effect on the Kondo state of a magnetic impurity, or a quantum dot, embedded in a two-dimensional (2D) system has received, in the last decade, renewed attention [8-13]. However, less attention has been given to an impurity coupled to a one-dimensional (1D) system [14,15]. Nevertheless, for either $1 \mathrm{D}$ or 2D, the effect SOC has on the Kondo regime became a controversial topic. Indeed, while some theory [15] and experimental [16] groups argue that SOC suppresses the Kondo effect, other groups arrived at the opposite conclusion, arguing that SOC enhances $T_{K}[10,12,14]$. Other studies predict that $T_{K}$ is not affected by SOC $[4,8]$, while still others concluded that the effect depends upon the parameters defining the system [9]. It is expected that in a 1D conductor, where SOC produces only forward and backward scattering, it should have a stronger influence than in 2D systems [14]. On one hand, Sousa et al. have concluded, using a renormalization group analysis, that SOC exponentially increases $T_{K}$ in 1D [14]. On the other hand, using the Hirsch-Fye Quantum Monte Carlo simulation and a slave-boson mean-field approximation, Chen and Han concluded that $T_{K}$ is reduced by SOC [15].

Here, we open a short parenthesis to mention that a related phenomenon, viz., Kondo effect in topological insulators (TI), bears some resemblances to the Kondo effect discussed here, but the list of differences - e.g., in TI we have absence of spin precession, no interband scattering, energy dependent density of states, etc. (see Ref. [17]) — is too large to warrant a direct comparison to our results.

The discussion above implies that the physics associated to the Kondo state under the effect of SOC requires a more profound understanding, comprising an effort of synthesis and clarification. Finally, most of the studies considered just SOC acting in the bulk, neglecting SOC acting at the impurity itself [18].

In this work, we show that the Haldane expression for $T_{K}[1,19,20]$, originally obtained for a zero-SOC single impurity Anderson model (SIAM), is still valid, with 
Kondo effect under the influence of spin-orbit coupling in a quantum wire

renormalized parameters, for a SIAM in which the Fermi sea, modeled as a 1D quantum wire (QW) has Rashba [21] and linear Dresselhaus [22] SOC, with couplings $\alpha$ and $\beta$, respectively. This occurs because the hybridization matrix, which incorporates the coupling between impurity and Fermi sea, and thus determines the Kondo state, is diagonal and spin independent (thus a scalar function) for both finite- and zero-SOC models. This may seem surprising, as SOC breaks full spin SU(2) symmetry. However, we argue that this property of the hybridization function is guaranteed, in our case, by time-reversal symmetry, which is preserved by SOC. To illustrate that, and to find analytical expressions for the renormalized parameters of the Haldane expression for $T_{K}$, we perform a change of basis that places the finite-SOC SIAM Hamiltonian into a form similar to that of the zero-SOC SIAM. This basis is then used to define a global pseudospin [23] wave-vector-dependent operator, obeying an SU(2) algebra, whose components (denoted by $\boldsymbol{S}_{Q}^{i}$ ) commute with the finite-SOC SIAM Hamiltonian for arbitrary values of $\alpha$ and $\beta$. This shows that the Hamiltonian has a pseudo-spin SU(2) symmetry, similar in spirit to the persistent spin helix (PSH) SU(2) symmetry found by Bernevig et al. [24] for a $2 \mathrm{D}$ model, with the restriction $\alpha=\beta$;

We then present numerical support to the analytical results described above by using the Numerical Renormalization Group (NRG) in two different regimes of the SIAM: (i) a wide-band regime, i.e., where the half band-width $D$ is much larger than the impurity Coulomb repulsion $U$ and its coupling to the band $\Delta$. In that case, the analytical results obtained through the appropriate Haldane expression for the Kondo temperature [see Eq. [37] in Ref. 19, reproduced below as Eq. (11)], are in excellent agreement with the NRG results, showing that, indeed, in this regime, SOC exponentially decreases the Kondo temperature; (ii) in an intermediate regime, where $U=D>\Delta$, with the Fermi energy close to the bottom of the band. The motivation for analyzing this regime is to compare the results obtained by Chen and Han [15], using Quantum Monte Carlo (QMC) calculations, with our NRG results. Finally, the analytical results for the Kondo temperature are compared to numerical results obtained by the Projector Operator Approach [25, 26] (POA), for yet a third regime, that of infinite Coulomb repulsion, and shown to be in excellent agreement.

\section{Model}

The system consists of a quantum wire (QW), i.e., a 1D Fermi sea (along the $x$-axis), coupled to a magnetic impurity. It is modeled by a SIAM [1,27] extension, modified to include Rashba [21] and linear Dresselhaus [22] SOC. The Hamiltonian is given by $H=H_{\text {wire }}+H_{\text {imp }}+H_{\text {hyb }}$, where

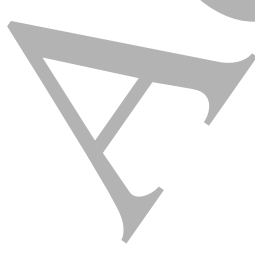

$$
\begin{aligned}
H_{w i r e}= & -\sum_{k, \sigma}(2 t \cos k-\mu) c_{k \sigma}^{\dagger} c_{k \sigma} \\
& -\sum_{k} 2 \sin k\left(\gamma c_{k \uparrow}^{\dagger} c_{k \downarrow}+\text { h.c. }\right),
\end{aligned}
$$


Kondo effect under the influence of spin-orbit coupling in a quantum wire
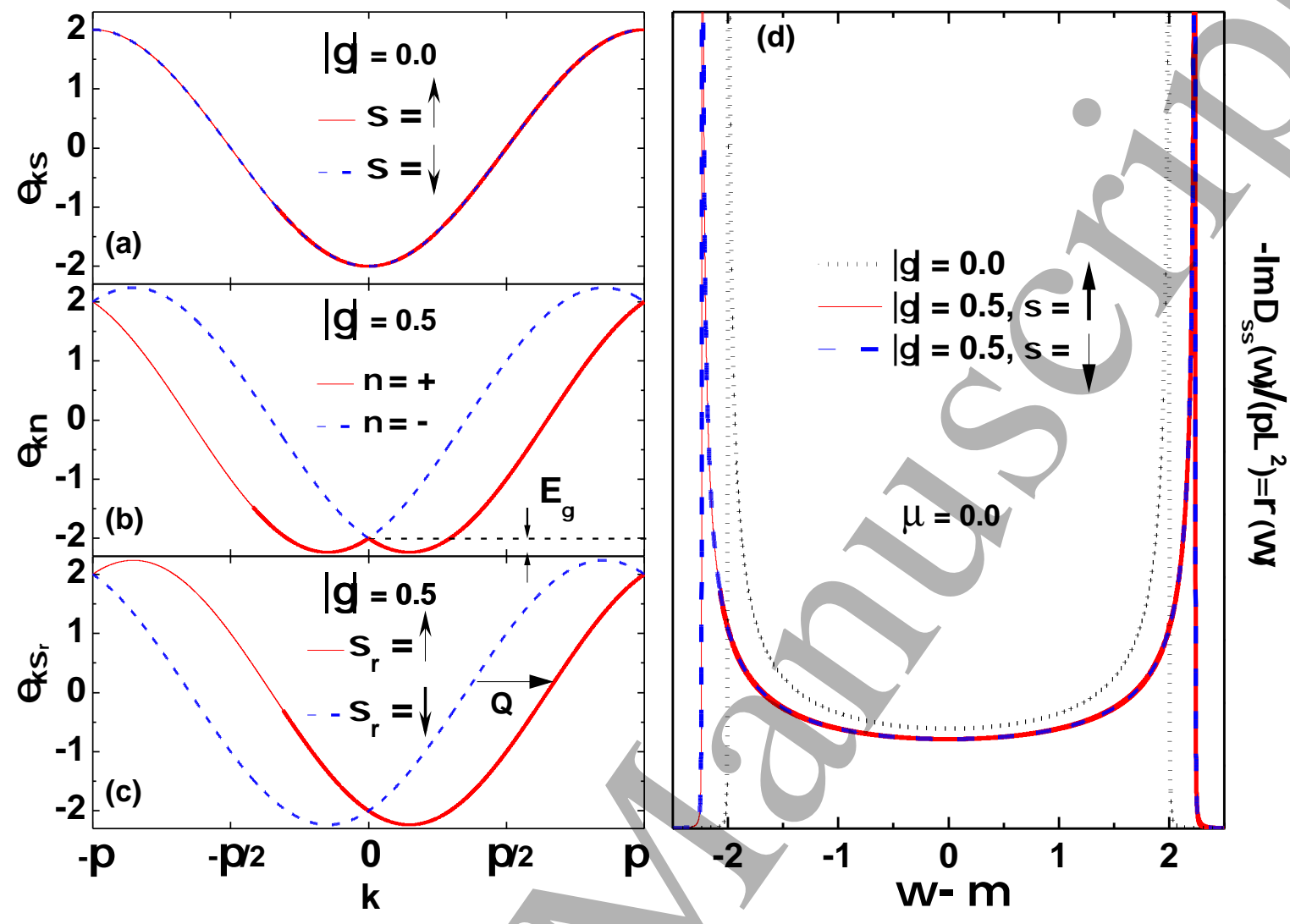

Figure 1. (a) Quantum wire dispersion $\epsilon_{k \sigma}+\mu$ for zero-SOC. Solid/dashed (red/blue) curve for $\sigma=\uparrow / \downarrow$ (b) Dispersion $\epsilon_{k \nu}$ for $|\gamma|=0.5$. Here, the bands are characterized by the helicity quantum number $\nu$ : solid/dashed (red/blue) curve for $\nu=+/-$ (c) Same as in (b), but now the bands are characterized by the quantum number $\sigma_{r}$ : solid/dashed (red/blue) curve for $\sigma_{r}=\uparrow / \downarrow$. Wave vector $Q$, connecting both bands, is discussed in the text. (d) $\operatorname{DOS} \rho(\omega)=-\operatorname{Im} \Delta_{\sigma \sigma}(\omega) /\left(\pi \Lambda^{2}\right)$ with and without SOC. The dotted black curve corresponds to zero-SOC $(|\gamma|=0)$ and the solid/dashed (red/blue) curves correspond to $|\gamma|=0.5$, for $\sigma=\uparrow / \downarrow$, for an arbitrary spin quantization axis. Note in panel (b) the definition of the SOC energy, $E_{\gamma}$, used in Fig. 3(a). All results are shown for $\mu=0$.

$$
\begin{aligned}
& H_{i m p}=\sum_{\sigma} \epsilon_{0} n_{0 \sigma}+\sum_{\sigma} \frac{U}{2} n_{0 \sigma} n_{0 \bar{\sigma}}, \\
& H_{h y b}=\sum_{k}\left[\sum_{\sigma}\left(V_{k} c_{k \sigma}^{\dagger} c_{0 \sigma}\right)+\right. \\
& \left.i \gamma_{i m p, k} c_{k \downarrow}^{\dagger} c_{0 \uparrow}+i \gamma_{i m p, k}^{*} c_{k \uparrow}^{\dagger} c_{0 \downarrow}+\text { h.c. }\right] .
\end{aligned}
$$

In the equations above, $c_{k \sigma}^{\dagger}\left(c_{k \sigma}\right)$ creates (annihilates) an electron with momentum $k$ and spin $\sigma$ in the QW, which is modeled by a 1D tight-binding approximation with nearest-neighbor hopping $t$, lattice parameter $a=1$, and $\mu=\epsilon_{F}$ is the chemical potential (Fermi energy). Note that $\mu$ will be set to 0 (half-filling) for most calculations, except 
when we investigate the Kondo state for a Fermi energy close to the bottom of the band, when it will be set to $\mu=\epsilon_{F}=-0.8$. The second term in Eq. (1) describes the SOC [28], where $\gamma=\beta+i \alpha, i=\sqrt{-1}$. The impurity is modeled by $H_{i m p}$, Eq. (2), where $\epsilon_{0}$ is the impurity orbital energy and $U$ is the Coulomb repulsion for its double occupancy. The operator $c_{0 \sigma}^{\dagger}\left(c_{0 \sigma}\right)$ creates (annihilates) an electron with spin $\sigma$ at the impurity, and $n_{0 \sigma}=c_{0 \sigma}^{\dagger} c_{0 \sigma}$. Equation (3) represents the coupling between the impurity and the QW, where the first term denotes the hybridization $V_{k}$, which preserves the electron spin between the impurity and the QW, while the other term represents the spin-flip coupling due to the local SOC at the impurity, of magnitude $\gamma_{i m p, k}[18,29]$. As it is the case for the hybridization $V_{k}, \gamma_{i m p, k}$ depends, in principle, upon the linear momentum $k$. However, for simplicity, we will assume that the two parameters are $k$-independent, denoted from now on as $V$ and $\gamma_{i m p}$. Note that, in the literature, a linear approximation of the electronic dispersion, valid for small values of $k[14,15]$, is frequently used. In the present study, we will not make this approximation, taking $k$ in the whole interval, i.e., $-\pi \leq k \leq \pi$. Finally, we note that the results shown in Figs. 1 and 4 were obtained for $t=1$ as the energy unit, while for the NRG results in Figs. 2 and 3 the half band-width $D=1$ was used as energy unit (thus, $t=1 / 2$ ).

\section{Spin rotation and SIAM Hamiltonian in the $\sigma_{r}$ basis}

Let us first analyze the decoupled (no impurity) QW. In Fig. 1(a), it is shown the dispersion when there is no SOC, $\gamma=0$ (solid/dashed (red/blue) curve for spin up/down). In this case, the Hamiltonian has spin $\mathrm{SU}(2)$ symmetry, i.e., the spin is a good quantum number and the energy dispersion does not depend upon it. Thus, the eigenstates $|k \uparrow\rangle$ and $|k \downarrow\rangle$ (in the $S_{z}$ basis) are degenerate and the z-axis can be chosen to point in any direction. However, for finite-SOC, the spin angular momentum, for an arbitrary quantization axis, is no longer a good quantum number, because of the spin-mixing term in Eq. (1). Nonetheless, as SOC preserves time-reversal symmetry, we can define a helicity operator $h$ such that $\left[h, H_{\text {wire }}\right]=0$, and whose eigenvalues $\nu= \pm$ are thus good quantum numbers for the eigenstates of $H_{\text {wire }}$. Indeed, in the helicity basis

$$
c_{k \nu}^{\dagger}=\frac{1}{\sqrt{2}}\left(c_{k \uparrow}^{\dagger}+\nu s_{k} e^{i \phi} c_{k \downarrow}^{\dagger}\right),
$$

where $\phi=\tan ^{-1}(\alpha / \beta)$ and $s_{k}=\operatorname{sgn}(k), H_{\text {wire }}=\sum_{k, \nu} \epsilon_{k \nu} c_{k \nu}^{\dagger} c_{k \nu}$ is diagonal, with a dispersion relation

$$
\epsilon_{k \nu}=-2 t \cos k-2 \nu|\gamma||\sin k|+\mu .
$$

Figure 1(b) shows $\epsilon_{k \nu}$, for $|\gamma|=0.5$, plotted as a function of $k$ for each $\nu$ : the lower band (solid (red) curve) associated to the quantum number $\nu=+$ and the upper band (dashed (blue) curve) to $\nu=-$. As SOC preserves time-reversal symmetry, i.e., $\left[\Theta, H_{\text {wire }}\right]=0$, where $\Theta$ is the time-reversal operator, we have degenerate Kramers doublets [30] in the same helicity band, $\epsilon_{k \nu}=\epsilon_{-k \nu}$. 
Kondo effect under the influence of spin-orbit coupling in a quantum wire

However, as the helicity $\nu$ is not defined for the impurity, its coupling to the QW mixes helicity channels and $\nu$ is not longer a good quantum number. We can rewrite $H_{\text {wire }}+H_{h y b}$ in a more convenient form by choosing another basis. The key here is to realize that the $|k \nu\rangle=c_{k \nu}^{\dagger}|0\rangle$ are eigenstates of the $S_{r}$ component of the spin angular momentum pointing along the direction $\hat{\boldsymbol{r}} \equiv[\theta=\pi / 2, \phi]$, for $s_{k}=+$, and along the opposite direction $-\hat{\boldsymbol{r}} \equiv[\theta=\pi / 2, \phi+\pi]$, for $s_{k}=-$. Thus, $\hat{\boldsymbol{r}}$ determines the direction of what is conventionally called the effective "spin-orbit magnetic field" [31], i.e., $\boldsymbol{B}_{\mathrm{SOC}}^{\mathrm{eff}}(k)=|\gamma| \sin (k) \hat{\boldsymbol{r}}$ [see Eq. (5)], such that when $k$ changes to $-k$ the effective magnetic field points in the opposite direction, thus conserving time-reversal symmetry. Note that, in this context, 'up' and 'down' refers to spin quantization 'along $\hat{\boldsymbol{r}}$ ', where $\hat{\boldsymbol{r}}$ lays on the $x y$ plane, somewhere in its first quadrant, depending on the ratio $\alpha / \beta$. Thus, as we will see next, for finite SOC, the presence of the impurity will make it advantageous to work in the $S_{r}$ basis, with spin quantum number $\sigma_{r}$.

The ground state of the Kondo regime is a singlet formed between the impurity and the conduction electrons, whose spins in the finite-SOC QW are good quantum numbers when quantized along the $\hat{\boldsymbol{r}}$ direction. As a consequence, it is natural to expect that it will be advantageous to choose a quantization axis along $\hat{\boldsymbol{r}}$ for the impurity as well. Thus, if we take $\hat{\boldsymbol{r}} \equiv[\theta=\pi / 2, \phi]$ as the spin quantization axis for the impurity, then the spin up $\left(\sigma_{r}=\uparrow \equiv+\right)$ and spin down $\left(\sigma_{r}=\downarrow \equiv-\right)$ impurity states are given by $c_{0 \sigma_{r}}^{\dagger}=1 / \sqrt{2}\left(c_{0 \uparrow}^{\dagger}+\sigma_{r} e^{i \phi} c_{0 \downarrow}^{\dagger}\right)$, where $c_{0 \sigma_{r}}^{\dagger}\left(c_{0 \sigma_{r}}\right)$ creates (annihilates) an electron at the impurity with spin $\sigma_{r}$, quantized along the $\hat{\boldsymbol{r}}$ direction, with the understanding that when $\sigma_{r}$ appears as a subscript it means $(\uparrow, \downarrow)$, and when it appears in an equation it means $(+,-)$, respectively.

The total Hamiltonian in this new basis is written as

$$
\begin{aligned}
H= & \sum_{k, \sigma_{r}} \epsilon_{k \sigma_{r}} c_{k \sigma_{r}}^{\dagger} c_{k \sigma_{r}}+\sum_{\sigma_{r}} \epsilon_{0} n_{0 \sigma_{r}}+\sum_{\sigma_{r}} \frac{U}{2} n_{0 \sigma_{r}} n_{0 \overline{\sigma_{r}}} \\
& +\sum_{k, \sigma_{r}} \Lambda\left(c_{k \sigma_{r}}^{\dagger} c_{0 \sigma_{r}}+c_{0 \sigma_{r}}^{\dagger} c_{k \sigma_{r}}\right),
\end{aligned}
$$

where $n_{0 \sigma_{r}}=c_{0 \sigma_{r}}^{\dagger} c_{0 \sigma_{r}}$ is the impurity number operator, $c_{k \sigma_{r}}^{\dagger}\left(c_{k \sigma_{r}}\right)$ creates (annihilates) an electron at the Fermi sea with momentum $k$ and spin $\sigma_{r}$ and $\Lambda=\left(V^{2}+\left|\gamma_{i m p}\right|^{2}\right)^{1 / 2}$, with a dispersion

$$
\epsilon_{k \sigma_{r}}=-2 \sqrt{t^{2}+|\gamma|^{2}} \cos \left(k-\sigma_{r} \varphi\right)+\mu,
$$

where $\varphi=\tan ^{-1}(|\gamma| / t)$. Each one of the bands in $\epsilon_{k \sigma_{r}}$, displaced from each other along the $k$-axis by $Q=2 \varphi$, is associated to one of the $S_{r}$ eigenvalues $\sigma_{r}=\uparrow, \downarrow$, as shown in Fig. 1(c).

\section{Time-reversal and the Hybridization Function}

It is well known that the hybridization function (which determines the properties of the Kondo state [32]) for the zero-SOC SIAM is a spin-independent scalar function, 
denoted by $\Delta(\omega)$. As the Hamiltonian shown in Eq. (6) is similar to that for the zeroSOC SIAM, one may be led to assume that this is still the case for the finite-SOC hybridization function. However, there is an important detail in Eq. (6): the QW dispersion $\epsilon_{k \sigma_{r}}$ is spin dependent [see Eq. (7) and Fig. 1(c)], which may imply that the hybridization function is spin dependent. A simple numerical calculation shows that this is not the case [see Fig. 1(d)]. A general argument shows that time-reversal symmetry requires the finite-SOC SIAM hybridization matrix $\tilde{\Delta}_{\sigma \sigma^{\prime}}(\omega)$ to be diagonal and spinindependent for any spin orientation $\sigma$ along an arbitrary quantization axis, like the one for the zero-SOC SIAM [32]. Indeed, the matrix elements of the $2 \times 2$ hybridization matrix can be written as $\tilde{\Delta}_{\sigma \sigma^{\prime}}(\omega)=\sum_{k} \Sigma_{\sigma \sigma^{\prime}}(k, \omega)$, where $\Sigma_{\sigma \sigma^{\prime}}(k, \omega)=\Lambda^{2} G_{\sigma \sigma^{\prime}}^{\text {wire }}(k, \omega)$, and $G_{\sigma \sigma^{\prime}}^{\text {wire }}(k, \omega)$ is the single-particle Green's function for the QW. An analysis of the expressions for the matrix elements $\Sigma_{\sigma \sigma^{\prime}}(k, \omega)$ indicates that their parity, in relation to $k$, can be readily obtained from Eq. (1): no-spin-flip terms $\Sigma_{\sigma \sigma}(k, \omega)$, produced by the first term in $H_{\text {wire }}$, which is associated to the kinetic energy $p^{2} / 2 m$, are therefore even in $k$ and spin independent, while non-diagonal spin-flip terms $\Sigma_{\sigma \bar{\sigma}}(k, \omega)$ are produced by the (SOC) second term in $H_{\text {wire, }}$ which, to preserve time-reversal symmetry, has to be odd in $k$. Thus, integrating $\Sigma_{\sigma \sigma^{\prime}}(k, \omega)$ in $k$ to obtain $\tilde{\Delta}_{\sigma \sigma^{\prime}}(\omega)$ results in $\tilde{\Delta}_{\uparrow \uparrow}(\omega)=\tilde{\Delta}_{\downarrow \downarrow}(\omega)=\tilde{\Delta}(\omega)$ and $\tilde{\Delta}_{\uparrow \downarrow}(\omega)=\tilde{\Delta}_{\downarrow \uparrow}(\omega)=0$. Therefore, as previously advertised, the spin independence of the hybridization function for the finite-SOC SIAM (despite the broken spin $\mathrm{SU}(2)$ symmetry) is guaranteed by the time-reversal symmetry. To illustrate these results, $-\operatorname{Im} \Delta_{\sigma \sigma}(\omega) /\left(\pi \Lambda^{2}\right)=\rho(\omega)$ is plotted in Fig. 1(d) for finiteSOC (solid/dashed (red/blue) curve for an arbitrary spin orientation $\sigma=\uparrow / \downarrow$ ), and, as expected, it does not depend upon the spin orientation.

\section{Pseudo-spin SU(2) Symmetry}

Motivated by the fact that the dispersion relation in Eq. (7) satisfies the identity $\epsilon_{k \sigma_{r}}=\epsilon_{k+Q \bar{\sigma}_{r}}$, which guarantees that a 2D Fermi sea has a pseudo-spin $\mathrm{SU}(2)$ symmetry (when $\alpha=\beta$ ) [24], we analyze if this is the case too for our system. To accomplish that, we generalize the problem to treat the Anderson model by introducing the spinor operators (in the $\sigma_{r}$ basis) $\boldsymbol{c}_{k Q}^{\dagger}=\left\{\begin{array}{ll}c_{k \uparrow}^{\dagger} & c_{k+Q \downarrow}^{\dagger}\end{array}\right\}$ and $\boldsymbol{c}_{0}^{\dagger}=\left\{\begin{array}{ll}c_{0 \uparrow}^{\dagger} & c_{0 \downarrow}^{\dagger}\end{array}\right\}$, and with them construct the operators $2 \boldsymbol{S}_{Q}^{i}=\sum_{k} \boldsymbol{c}_{k Q}^{\dagger} \boldsymbol{\sigma}^{i} \boldsymbol{c}_{k Q}+\boldsymbol{c}_{0}^{\dagger} \boldsymbol{\sigma}^{i} \boldsymbol{c}_{0}$, where the $\boldsymbol{\sigma}^{i}$ are the Pauli matrices and $Q=2 \varphi$ [see Eq. (7)]. These operators obey the angular momentum commutation relations $\left[S_{Q}^{i}, S_{Q}^{j}\right]=i \varepsilon^{i j l} S_{Q}^{l}$, where $\varepsilon^{i j l}$ is the Levi-Civita symbol. It can be shown that the $S_{Q}^{i}$ (for $\left.i=x, y, z\right)$ commute with the finite-SOC SIAM Hamiltonian. Note that, as in Ref. [24], the commutation $\left[S_{Q}^{i}, H\right]=0$ is satisfied because of the equality $\epsilon_{k+Q \sigma_{r}}=\epsilon_{k \bar{\sigma}_{r}}$, when $Q=2 \varphi$ [see Eq. (7) and Fig. 1(c)] showing that our Hamiltonian is pseudo-spin $\mathrm{SU}(2)$ symmetric. Thus, the operators $S_{Q}^{i}$ are the generators of the symmetry operations connected to the PSH states [24], which the authors believe may be associated with the structure of the Kondo cloud formed in our system, as observed in a previous work on Topological Insulators (see Fig. 10(a) in Ref. [33]). 
Kondo effect under the influence of spin-orbit coupling in a quantum wire

\section{Renormalized Haldane Expression}

Through the application of Anderson's poor man's scaling approach [34] to the SIAM, different expressions for the Kondo temperature $T_{K}$ can be found for a variety of parameter regimes [1]. All of them, collectively known as Haldane expression [19,20], are proportional to an exponential $\exp \left(\pi \epsilon_{0}\left(\epsilon_{0}+U\right) / 2 \Delta U\right)$ multiplied by a function of $\Delta, D$, $\epsilon_{0}$ and $U$, whose form depends on these parameters relative values. In the wide-band limit, i.e., $D \gg U, \Delta,\left|\epsilon_{0}\right|$, Haldane obtained (see Eq. [37] in Ref. 19)

$$
T_{K}=0.364(2 \Delta U / \pi)^{\frac{1}{2}} \exp \left[\frac{\epsilon_{0}\left(\epsilon_{0}+U\right)}{2 \Delta U / \pi}\right],
$$

where $\Delta=\pi V^{2} \rho\left(\epsilon_{F}\right)$ and $\rho\left(\epsilon_{F}\right)$ is the band DOS at the Fermi energy $\epsilon_{F}$. As our Hamiltonian [Eq. (6)] is formally equivalent to the zero-SOC SIAM Hamiltonian used to obtain Eq. (8), the Haldane expression should be valid for our finite-SOC SIAM as well, but with band parameters renormalized by SOC (an SOC renormalized parameter will be denoted with a $\sim$ on top of it). Indeed, as illustrated in Fig. 1(d), which compares the DOS for $\gamma=0.0$ (dotted black line) with that for $|\gamma|=0.5$ (solid/dashed (red/blue) for $\sigma=\uparrow / \downarrow)$, the bulk SOC $\gamma$ increases the bandwidth and thus decreases the DOS $\tilde{\rho}\left(\epsilon_{F}\right)$, at $\epsilon_{F}=0$. Thanks to the analytical expression for $\epsilon_{k \sigma_{r}}$ [Eq. (7)], obtained through the spin rotation, we derive simple analytical expressions for the renormalized semi-bandwidth

$$
\tilde{D}=2 \sqrt{t^{2}+|\gamma|^{2}}
$$

as well as for the band DOS at the Fermi energy $\tilde{\rho}(0)=1 /\left(2 \pi \sqrt{t^{2}+|\gamma|^{2}}\right)$. Therefore, the renormalized hybridization function at half filling $\tilde{\Delta}=\tilde{\Delta}(0)$ can be written as

$$
\tilde{\Delta}=\pi \Lambda^{2} \tilde{\rho}(0)=\frac{V^{2}+\left|\gamma_{i m p}\right|^{2}}{2 \sqrt{t^{2}+|\gamma|^{2}}} .
$$

Finally, replacing $\tilde{\Delta}$ for $\Delta$ in Eq. (8), the renormalized finite-SOC SIAM Kondo temperature $\tilde{T}_{K}$, in the wide-band limit, is given by

$$
\tilde{T}_{K}=0.364(2 \tilde{\Delta} U / \pi)^{\frac{1}{2}} \exp \left[\frac{\epsilon_{0}\left(\epsilon_{0}+U\right)}{2 \tilde{\Delta} U / \pi}\right] .
$$

In particular, if one takes the $U \rightarrow \infty$ limit, one obtains [1]

$$
\tilde{T}_{K} \propto \sqrt{\tilde{\Delta} \tilde{D}} \exp \left(\pi \epsilon_{0} / 2 \tilde{\Delta}\right)
$$

where it should be noticed that, differently from the wide-band limit [Eq. (11)], the multiplieative constant in Eq. (12) is unknown. Through the renormalized Haldane expressions given in Eqs. (11) and (12), one can easily analyze the SOC impact on the Kondo temperature. The prefactor in the infinite- $U$ regime depends only on the local SOC term $\left(\gamma_{i m p}\right)$ and does not depend on the bulk SOC $(\gamma)$. However, in the $D \gg U$ regime, the prefactor depends upon both parameters $\left(\gamma_{i m p}\right.$ and $\left.\gamma\right)$. We do not attribute 
Kondo effect under the influence of spin-orbit coupling in a quantum wire

any specific relevance to this difference in behavior between the two prefactors, as it does not impact the universality of $T_{K}$, whose dominant dependence is given by the exponential term shown in Eqs. (11) and (12). In addition (keeping in mind that, in the Kondo regime, $\epsilon_{0}<\mu$, since $\epsilon_{F}=\mu$ ), Eq. (10), for the renormalized hybridization $\tilde{\Delta}$, implies that the local SOC $\gamma_{i m p}$, by increasing $\tilde{\Delta}$, exponentially increases $\tilde{T}_{K}$, while the bulk SOC term $\gamma$ exponentially decreases it, by decreasing $\tilde{\Delta}$.

Next, we will numerically validate the analytical results obtained for the wide-band regime (using NRG) and for the infinite- $U$ regime (using POA). In addition, we will compare our NRG results with the QMC results in Ref. 15, for the intermediate regime, $U=D>\Delta$, with the Fermi energy close to the bottom of the band.

\section{Numerical Results}

\subsection{Numerical Renormalization Group Results}

In Fig. 2, we show Kondo temperature results for finite-SOC, $\tilde{T}_{K}$ (in $\log _{10}$ scale), as a function of $U / \tilde{\Delta}$, for $U=1.0 \times 10^{-3}, V /=5.5 \times 10^{-3}, 0.0 \leq|\gamma| \leq 0.5, \gamma_{i m p}=0.0$, $\mu=0.0$, at the particle-hole symmetric point $\epsilon_{0}-\mu=-U / 2$. The (red) squares curve was obtained using NRG, while the (blue) circles curve was obtained analytically through Eq. (11). We used this set of parameters for two reasons: first, the wide-band limit, i.e., $D \gg U, \tilde{\Delta},\left|\epsilon_{0}\right|$, allows for a very precise determination of the prefactor to the exponential [see Eq. (11)]. Thus, in this regime, the Haldane expression is supposed to be the most accurate. This can be confirmed by its very good agreement with NRG, as shown in the figure. Second, for $U \gg \tilde{\Delta}$, and in the particle-hole symmetric point $\epsilon_{0}-\mu=-U / 2$ and $\mu=0$, one is deep into the Kondo regime, therefore, NRG is probing the properties of the SIAM very close to its strong coupling fixed point. The very good agreement shown by the results in Fig. 2 imply that the SOC-induced reduction of the Kondo temperature is directly tied to the suppression of the hybridization $\tilde{\Delta}$ at the Fermi energy, which is caused by the widening of the band [see Fig. 1(d)], and this effect is very accurately described by the Haldane expression, Eq. (11), giving strong support to the analytical results presented in the previous section.

The situation is more involved for the second regime we analyzed, which we call intermediate regime, i.e., $U=D>\Delta$, where, in addition, we have moved the Fermi energy close to the bottom of the band. Recent results [15], for a model very similar to ours, obtained using QMC, have reported a polynomial dependence of the Kondo temperature with SOC for this intermediate regime (see Fig. 3(a) in Ref. 15). Their conclusion is similar to the one we obtained for the wide-band regime, namely, that the reduction of the hybridization at the Fermi energy, caused by SOC, is responsible for the decrease in the Kondo temperature. In Fig. 3(a), to compare our NRG results with the QMC ones in Ref. 15, it is shown $\tilde{T}_{K}\left(E_{\gamma}\right) / T_{K}(0)$ vs $E_{\gamma}$ [where $E_{\gamma}=2\left[\sqrt{t^{2}+|\gamma|^{2}}-t\right]$ is the so-called SOC energy, indicated in Fig. 1(b)], for $U=1.0, V=0.396,0.0 \leq|\gamma| \leq 0.5$, $\gamma_{i m p}=0.0, \mu=-0.8$, and two different values of $\epsilon_{0}-\mu=-0.3$ [(red) squares curve] 
and -0.7 [(blue) circles curve]. For this value of chemical potential, the Fermi energy is just 0.2 above the bottom of the band. The contrast to the results shown in Fig. 2 is striking. Note that we have plotted (not shown) the two curves in panel (a) in $\log _{10}$ scale for the vertical axis (vs $U / \tilde{\Delta})$ and the behavior is clearly not Haldane-like. In Fig. 3(b), we show NRG results for $\tilde{T}_{K}(\gamma) / T_{K}(0)$ vs $U / \tilde{\Delta}$ for the same parameters as in Fig. 3(a), but for $\mu=0.0$ (i.e., at half-filling), using a $\log _{10}$ scale. It is very clear that, for the particle-hole symmetric point, contrary to what happens when the Fermi energy is close to the bottom of the band [Fig. $3(\mathrm{~b})], \tilde{T}_{K}(\gamma) / T_{K}(0)$, plotted against $U / \tilde{\Delta}$, shows a very-closeto exponential behavior. We do not plot results for the Haldane expression [Eq. (11)] because, as already mentioned above, that expression compares well with the NRG results just for the wide-band limit [35]. Nonetheless, the contrast between the results at half-filling $[\mu=0.0$, panel (b)] and those for the Fermi energy close to the bottom 
1

2

3

4

5

6

7

8

9

Kondo effect under the influence of spin-orbit coupling in a quantum wire

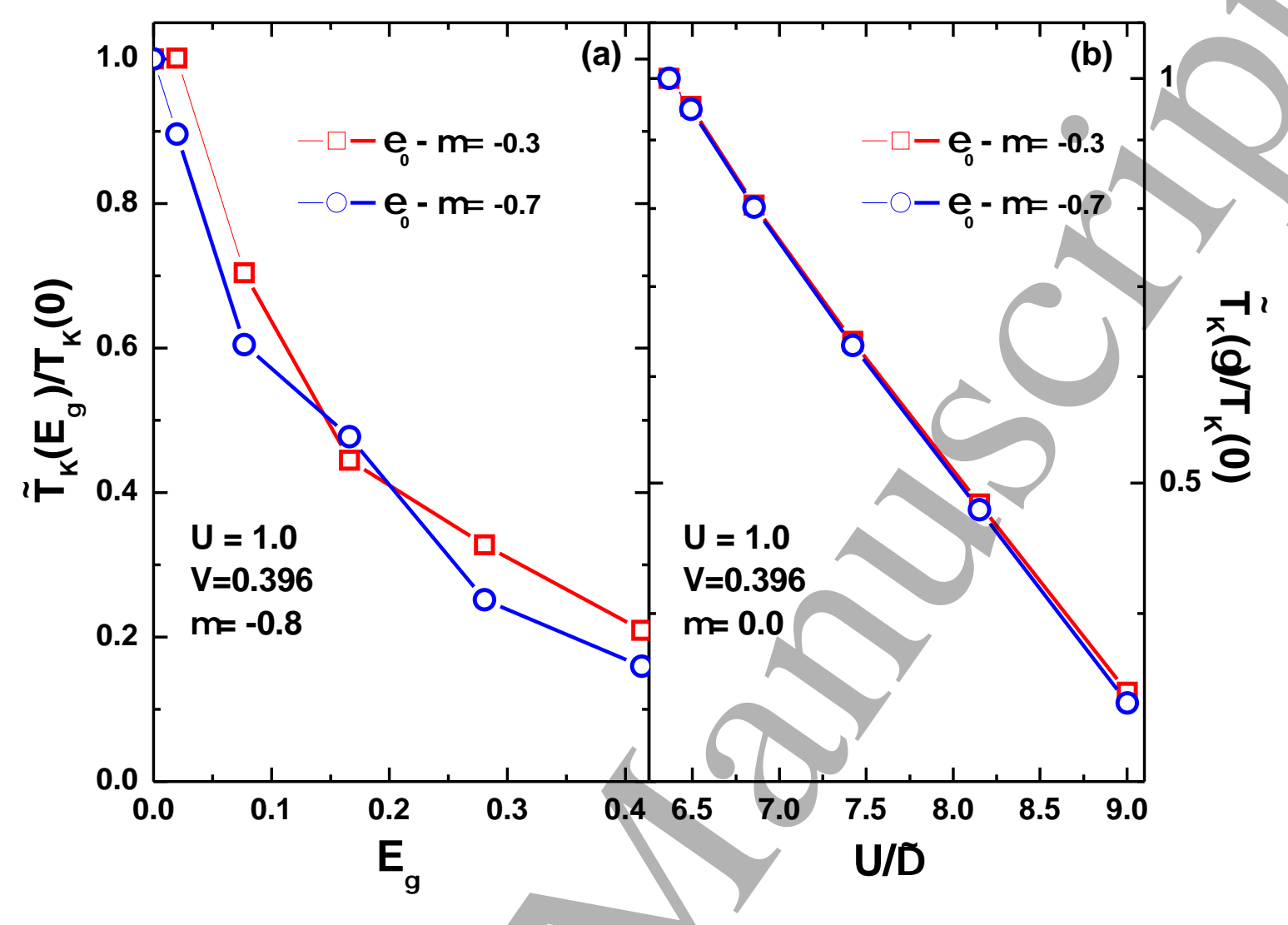

Figure 3. (a) NRG results for $\tilde{T}_{K}\left(E_{\gamma}\right) / T_{K}(0)$ vs $E_{\gamma}$ (SOC energy, see main text) in the intermediate regime, for $U=1.0, V=0.396,0.0 \leq|\gamma| \leq 0.5, \gamma_{i m p}=0.0, \mu=-0.8$ (thus, the Fermi energy is 0.2 above the bottom of the band), and two different values of $\epsilon_{0}-\mu:-0.3$ [(red) squares curve] and -0.7 [(blue) circles curve]. (b) Same parameters as in (a), except for the chemical potential, now at half-filling $(\mu=0.0)$. In addition, the horizontal axis is now $U / \bar{\Delta}$, instead of $E_{\gamma}$, and the vertical axis is in $\log _{10}$ scale, showing that the Kondo temperature has an almost exponential behavior dependence on $U / \tilde{\Delta}$, similar to the results for the wide-band limit, Fig. 2.

of the band $[\mu=-0.8$, panel (a)], indicates that the polynomial behavior reported in Ref. 15 is caused by the proximity of the Fermi energy, and thus the Kondo peak, to the singularity at the bottom of the band. Indeed, as $\gamma$ changes, the singularity moves [see Fig. 1(d)], altering its effect over the impurity's local density of states (LDOS), thus over its Kondo peak, and, by extension, over its Kondo temperature. A similar effect was observed for a related 2D model [9,36]. Indeed, as shown in detail in Ref. 9 [see its Fig. (2)], for $U=D$ and with the Fermi energy sitting close to the bottom of the band, as it is the case for the intermediate regime analyzed here and in Ref. 15, the broad $\epsilon_{0}-\mu$ peak in the impurity's LDOS is strongly affected by the singularity at the bottom of the band, and this has an effect on the width of the Kondo peak, thus in the associated Kondo temperature, resulting in the behavior seen in Fig 3(a). None of that is seen in Fig. 2 and very little of it in Fig. 3(b), because both the Kondo and the $\epsilon_{0}-\mu$ peaks are far from the singularity and are not affected by its movement (more so in the case of the results in Fig. 2, where, in addition, $U \ll D$ ). It is not noticeable in 
Fig. 3(b), but the first two points $(|\gamma|=0$ and 0.1$)$, for both values of $\epsilon_{0}$, slightly deviate from the exponential behavior followed by the rest of the points (at higher $|\gamma|$ values). This is consistent with our interpretation of the non-exponential behavior present for $\mu=-0.8$ being caused by the proximity of the bottom of the band singularity to the Kondo peak, as the singularity moves away from it as SOC increases.

Thus, in summary, the QMC results in Ref. 15, reporting a polynomial behavior of the Kondo temperature with SOC, in qualitative agreement with our results obtained through NRG [see Fig. 3(a)], do not contradict our main conclusion regarding the suitability of using Haldane's expression [Eq. (11)] to understand the SOC effect on the Kondo regime. The reason is clear: the influence of any structures in the hybridization function (like the singularity at its bottom), which only manifests itself in the very specific regime analyzed in Fig. 3(a), where the Fermi energy is close to the bottom of the band and $U=D$, is irrelevant in the wide-band (or flat-band) regime, $\mu=0.0$ and $D \gg U, \Delta,\left|\epsilon_{0}\right|$, for which Eq. (11) was derived. Finally, we agree with Chen and Han [15] in their assessment that the poor man's scaling results [14], pointing to an exponential increase of $T_{K}$ with SOC in a $1 \mathrm{D}$ system similar to ours, is a high temperature effect, which does not describe the properties of the Kondo ground state under the influence of SOC, at least in regards to the Kondo temperature.

The NRG approach was performed using Wilson's discretization parameter set to $\Lambda=2.0,2000$ many-body states were kept after each NRG iteration (except for the calculations near the bottom of the band, where it was necessary to keep 20000), and we made use of the $z$-trick averaging in the discretization procedure. In addition, the Kondo temperature was obtained through Wilson's criterion [1]. We have used the NRG Ljubljana open source code [37] for all NRG calculations.

\subsection{Projector Operator Approach Results}

In order to test the analytical results in the infinite- $U$ regime, we use POA, which is a numerical technique that works quite well in this regime [25, 26]. We find the parameter dependence of $\tilde{T}_{K}$ by calculating the zero-temperature finite-SOC impurity magnetic susceptibility, $\tilde{\chi}_{i m p}$. It is obtained by numerically evaluating the impurity magnetization, $M_{i m p}=n_{0 \uparrow}-n_{0 \downarrow}$, due to the application of a vanishing external magnetic field $B$, coupled just to the impurity and oriented along $\hat{\boldsymbol{r}}$. It is well established that, in the strong coupling regime, $\tilde{\chi}_{i m p}(B=0)=\eta{\tilde{T_{K}}}^{-1}$, where $\eta$ is a constant [1]. Thus, $\tilde{T}_{K}$ can be obtained (but for a multiplicative constant) through the numerical calculation of $\tilde{\chi}_{i m p}$ for a vanishing magnetic field. The objective of these numerical calculations is to confirm Eq. (12).

Figure 4 shows a log-linear plot of $U \rightarrow \infty$ POA results for $\tilde{\chi}_{i m p}^{-1} / \sqrt{\tilde{\Delta} \tilde{D}}$ vs $\epsilon_{0} / \tilde{\Delta}$, either by varying $-0.17<\epsilon_{0}-\mu<-0.10$ (for $\mu=0$ ) and keeping $\tilde{\Delta}=0.029$ fixed (red squares), or for different values of $\tilde{\Delta}$, obtained by varying $0.10 \leq|\gamma| \leq 0.30$ and $0.23 \leq \Lambda \leq 0.30$, and keeping $\epsilon_{0}-\mu=-0.15$ constant (blue triangles). When $\tilde{\Delta}$ and $\tilde{D}$ are varied, an exponential behavior is only obtained after dividing $\tilde{\chi}_{i m p}^{-1}$ by $\sqrt{\tilde{\Delta} \tilde{D}}$, 


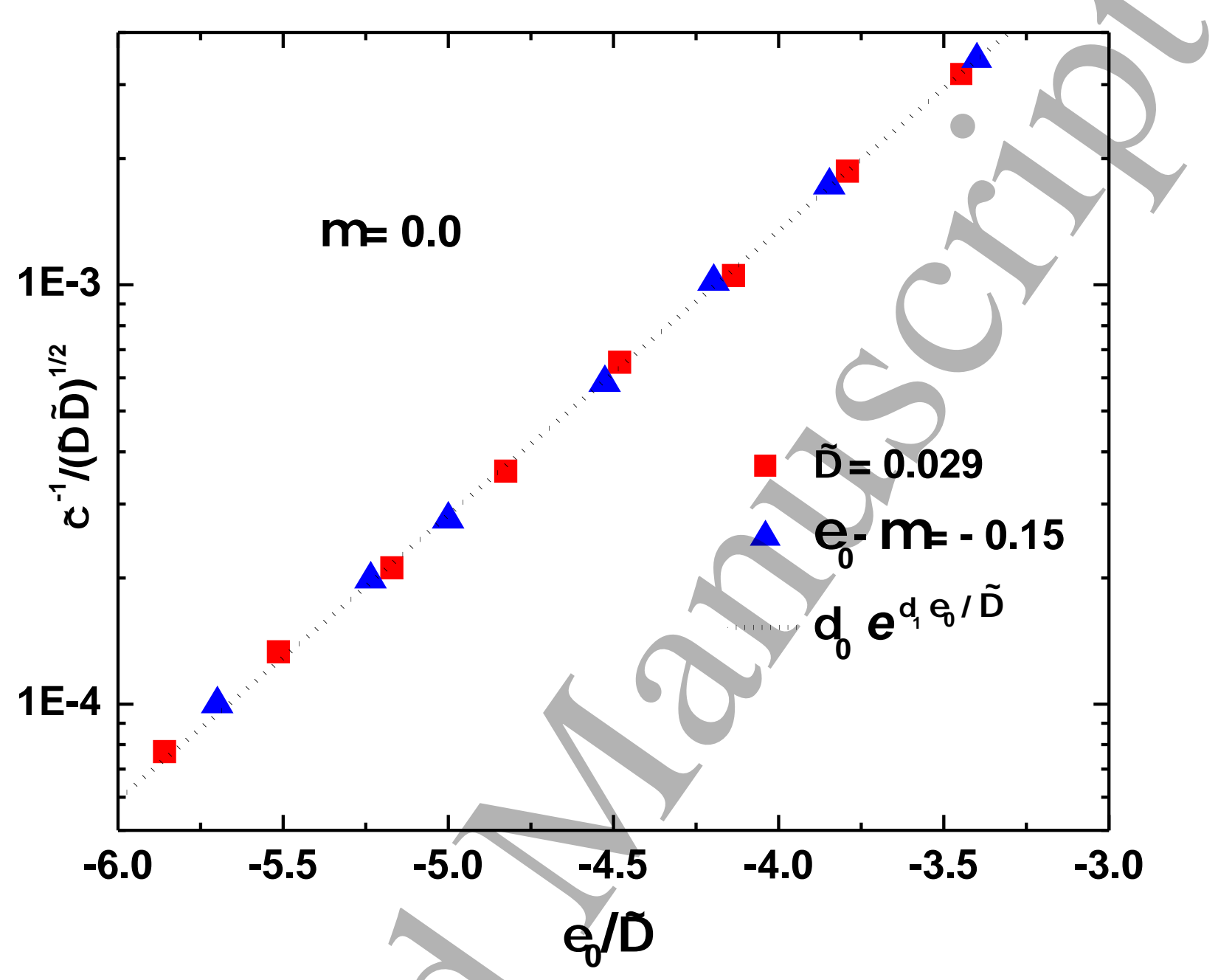

Figure 4. POA results for $\tilde{\chi}_{i m p}^{-1} / \sqrt{\tilde{\Delta} \tilde{D}}$ as a function of $\epsilon_{0} / \tilde{\Delta}$. The (red) squares curve was obtained by varying $-0.17<\epsilon_{0}-\mu<-0.10$, for $\mu=0$, and keeping $\tilde{\Delta}=0.029$ fixed, while the (blue) triangles were obtained by varying $\tilde{\Delta}$, by varying simultaneously $0.10 \leq|\gamma| \leq 0.30$ and $0.23 \leq \Lambda \leq 0.30$, and keeping $\epsilon_{0}-\mu=-0.15$ constant. The dotted black line is a fitting of the data using $\delta_{0} \exp \left(\delta_{1} \epsilon_{0} / \tilde{\Delta}\right)$, resulting in $\delta_{1}=\pi / 2$, with $\lesssim 1 \%$ error, and $\delta_{0}=0.699(\lesssim 3 \%)$.

confirming the prefactor dependence shown in Eq. (12). The dotted (black) line is an exponential fit of the data using $\delta_{0} \exp \left(\delta_{1} \epsilon_{0} / \tilde{\Delta}\right)$, resulting in $\delta_{1}=\pi / 2$ with $\lesssim 1 \%$ error, and $\delta_{0}=0.699(\lesssim 3 \%)$. Therefore, Fig. 4 shows that $\tilde{T}_{K}$ depends exponentially on $\pi \epsilon_{0} / 2 \tilde{\Delta}$, thus corroborating Eq. (12).

\section{Summary and conclusions}

In conclusion, we have shown, through a physically motivated change of basis, that the 1D finite-SOC SIAM Hamiltonian is similar to that for the zero-SOC SIAM. The form of the 1D finite-SOC SIAM Hamiltonian (for both the $S_{z}$ and helicity bases) seems to be inappropriate to deal with Kondo physics, since conduction channels with 
opposite quantum numbers (either $\sigma=\uparrow \downarrow$ or $\nu= \pm$ ) are mixed (either by SOC, or by the impurity itself). This issue can be circumvented if one exploits the fact that timereversal symmetry is not broken. Indeed, it is the time-reversal symmetry that renders the finite-SOC hybridization matrix diagonal and spin-independent (in any spin basis), thus a scalar, like the zero-SOC hybridization function. This can be seen in a more clear way once both the impurity and the conduction electrons are rotated to the $\sigma_{r}$ basis, where it becomes clear that the spin channels are not mixed neither by SOC, nor by the impurity, allowing a simple analytical treatment of the renormalized $\tilde{T}_{K}$, through the use of the Haldane expression, which is corroborated by NRG calculations in the wide-band regime $\left(D \gg U, \Delta,\left|\epsilon_{0}\right|\right)$ and by POA in the infinite- $U$ regime. In addition, NRG results for the intermediate regime $(U=D>\Delta$, with Fermi energy close to the bottom of the band), in qualitative agreement with QMC results presented in Ref. 15, indicate that it is the proximity of the Fermi energy to the structure at the bottom of the hybridization function (a singularity) that causes the divergence of the results from what one expects from Haldane's expression. Finally, it is shown that the 1D SOC-SIAM Hamiltonian, for arbitrary values of $\alpha$ and $\beta$, has a PSH SU(2) symmetry, in contrast to the 2D SOC Fermi sea, where the PSH state is restricted to the $\alpha=\beta$ case.

As to a possible experimental verification of our results, we note that it has been demonstrated recently the possibility of controlling the Rashba SOC in InAs nanowires through the use of a combination of metallic gates [38]. InAs nanowires have very strong Rashba SOC, whose strength can be further increased (more than doubled) by the above mentioned technique. The use of a system like that, coupled to either a quantum dot or an adsorbed magnetic impurity could be used to detect the change in $T_{K}$ caused by the Rashba SOC variation due to the electrostatic fields created by the gates.

The authors hope that the results presented here, as well as the conceptual insights, will be helpful in clarifying the somewhat confusing state of the properties of the Kondo regime of a quantum impurity coupled to a bath subjected to SOC.

\section{Acknowledgments}

It is a pleasure to acknowledge discussions with E. Vernek, G.J. Ferreira, and M.S. Figueira. VL acknowledges a PhD scholarship from the Brazilian agency Conselho Nacional de Desenvolvimento Científico e Tecnológico (CNPq), process 160071/2015-1, and financial support from the Generalitat Valenciana through grant reference Prometeo 2017/139. MM acknowledges a PhD scholarship from the Brazilian agency Coordenação de Aperfeiçoamento de Pessoal de Nível Superior (CAPES). GBM acknowledges financial support from CNPq, processes 424711/2018-4 and 305150/2017-0. EVA acknowledges financial support from CNPq, process 306000/2017-2.

\section{References}

[1] Hewson A C 1993 The Kondo Problem to Heavy Fermions (Cambridge University Press) URL http://dx.doi.org/10.1017/CB09780511470752 
[2] De Haas W J and Van Den Berg G J 1936 Physica 3440

[3] Kondo J 1964 Progress of Theoretical http://dx.doi.org/10.1143/PTP.32.37

[4] Meir Y and Wingreen N S 1994 Phys. Rev. B 504947

[5] Žutić I, Fabian J and Das Sarma S 2004 Rev. Mod. Phys. 76323

[6] Bader S and Parkin S 2010 Annu. Rev. Condens. Matter Phys. 171

[7] Datta S and Das B 1990 Applied Physics Letters http://dx.doi.org/10.1063/1.102730

[8] Malecki J 2007 Journal of Statistical Physics 129 741-757 ISSN $1572-9613$ URL https://doi.org/10.1007/s10955-007-9414-x

[9] Žitko $\mathrm{R}$ and Bonča J $2011 \quad$ Phys. Rev. B $\quad \mathbf{8 4 ( 1 9 )} 193411$ URL https://link.aps.org/doi/10.1103/PhysRevB.84.193411

[10] Zarea M, Ulloa S E and Sandler N 2012 Phys. Rev Lett. 108(4) 046601 URL https://link.aps.org/doi/10.1103/PhysRevLett.108.046601

[11] Mastrogiuseppe D, Wong A, Ingersent K, Ulloa S E and Sandler N 2014 Phys. Rev. B 90(3) 035426 URL https://link.aps .org/doi/10.1103/PhysRevB.90.035426

[12] Wong A, Ulloa S E, Sandler N and Ingersent K 2016 Phys. Rev. B 93(7) 075148 URL https://link.aps.org/doi/10.1103/PhysRevB.93.075148

[13] Chen L, Sun J, Tang H K and Lin H Q 2016 J. Phys. Condens, Matter 28396005

[14] de Sousa G R, Silva J F and Vernek E 2016 Phys. Rev. B 94(12) 125115 URL https://link.aps.org/doi/10.1103/PhysRevB.94.125115

[15] Chen L and Han R S 2017 ArXiv e-prints (Preprint 1711.05505)

[16] Gainon D and Heeger A J 1969 Phys. Rev. Lett. 22(26) 1420-1423 URL https://link.aps.org/doi/10.1103/PhysRevLett.22.1420

[17] Wang J and Culcer D 2013 Phys. Rev. B 88125140

[18] López R, Sánchez D and Serra L 2007 Phys. Rev. B $\quad \mathbf{7 6 ( 3 )} 035307$ URL https://link.aps.org/doi/10.1103/PhysRevB.76.035307

[19] Haldane F D M 1978 J. Phys. C: Sol. State Phys. 115015

[20] Haldane F D M 1978 Phys. Rev. Lett. 40416 and Phys. Rev. Lett. 40, 911 (1978)

[21] Bychkov Y A and Rashba E I 1984 Journal of Physics C: Solid State Physics 176039 URL http: //stacks.iop.org/0022-3719/17/i=33/a=015

[22] Dresselhaus G 1955 Phys. Rev.100(2) 580-586 URL https://link.aps.org/doi/10.1103/PhysRev. 100.580

[23] Winkler R and Zülicke U 2010 Physics Letters A 3744003

[24] Bernevig B A, Orenstein J and Zhang S C 2006 Phys. Rev. Lett. 97236601

[25] Roura-Bas P, Hamad I J and Anda E V 2015 Phys. Status Solidi (b) 252 421-430 URL http://dx.doi.org/10.1002/pssb.201451520

[26] Lopes V, Padilla R A, Martins G B and Anda E V 2017 Phys. Rev. B 95245133

[27] Anderson P W 1961 Phys. Rev. 124(1) 41-53 URL https : //link.aps .org/doi/10.1103/PhysRev. 124.41

[28] Mireles F and Kirczenow G 2001 Phys. Rev. B 64(2) 024426 URL https://link.aps.org/doi/10.1103/PhysRevB.64.024426

[29] Sun Q f, Wang J and Guo H 2005 Phys. Rev. B 71(16) 165310 URL https://link.aps.org/doi/10.1103/PhysRevB.71.165310

[30] Sakurai J J 1994 Modern Quantum Mechanics (Addison-Wesley)

[31] Manchon A, Koo H C, Nitta J, Frolov S M and Duine R A 2015 Nat. Mater. 14871

[32] Bulla R, Costi T A and Pruschke T 2008 Rev. Mod. Phys. 80395

[33] Allerdt A, Feiguin A E and Martins G B 2017 Phys. Rev. B 96035109

[34] Anderson P W 1970 J. Phys. C: Sol. State Phys. 32436

[35] It should be noted, as already mentioned at the beginning of Sec. 6, and explained in detail in Sec. 3.4 in Ref. 1, that other approximate analytical expressions for $T_{K}$ can be obtained for different regimes, but the intermediate regime is not one of them. Note that the three curves in Fig. 3(b) deviate slightly (not shown) from straight lines, most certainly because of a $U, \tilde{\Delta}$, and 
Kondo effect under the influence of spin-orbit coupling in a quantum wire

$\tilde{D}$ dependent prefactor. In addition, it should be also noted that for none of these expressions a numerical multiplicative factor, like the 0.364 in Eq. (11), has ever been obtained for any regimes other than the wide-band regime [1].

[36] Note that the effect of SOC over the hybridization function in 2D is restricted to the region close to the bottom of the band (see Fig. 4(b) in Ref. 13), while, in 1D, the SOC effect is two-fold: it suppresses the hybridization function at all energies, as well as it moves the singularity at the bottom of the band to lower energies.

[37] R. Žitko, NRG Ljubljana - open source NRG code available at http://nrgljubljana.ijs.si.

[38] Scherübl Z, Fülöp G m H, Madsen M H, Nygård J and Csonka S 2016 Phys. Rev. B 94035444

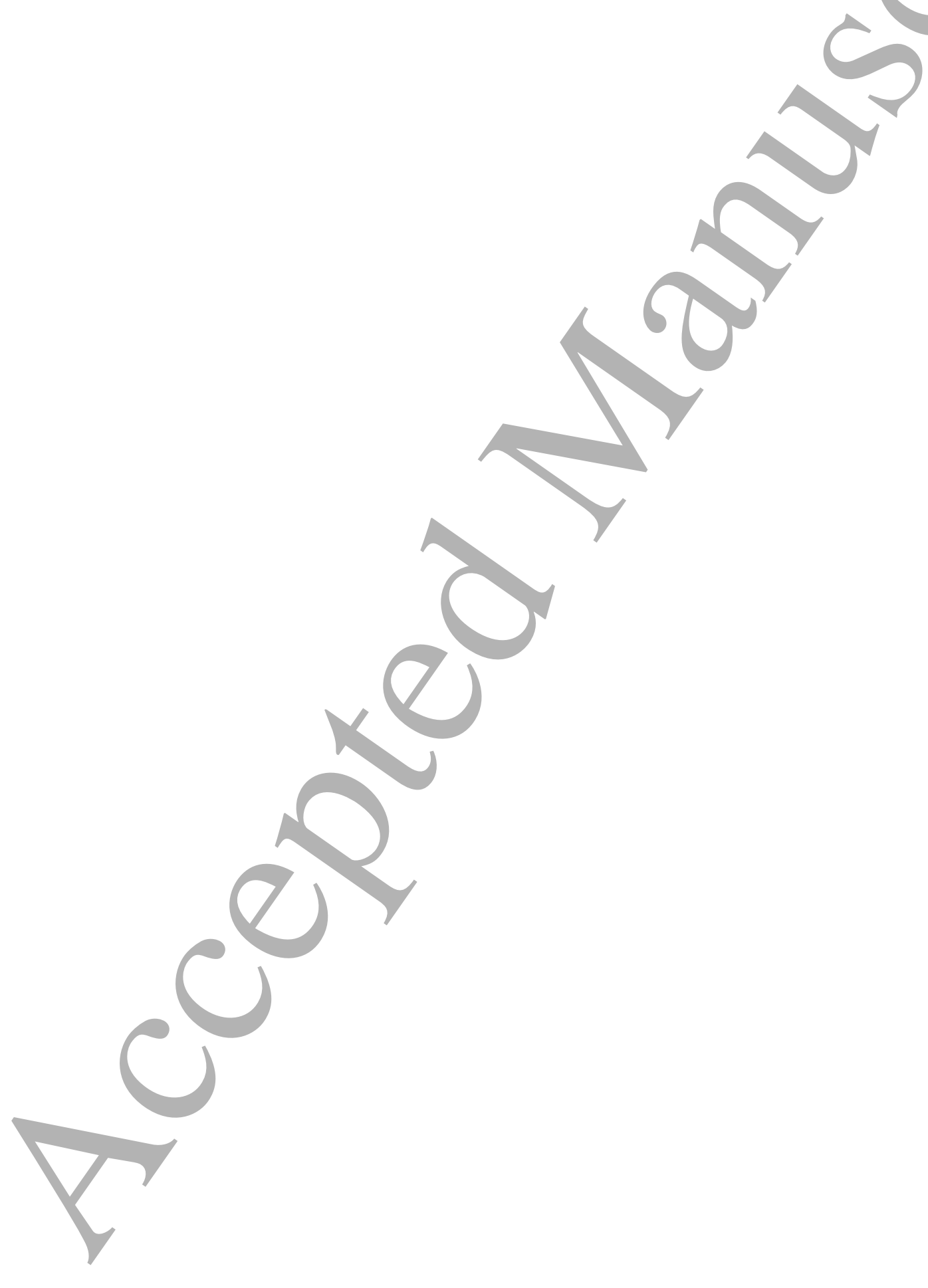

